albumin levels after two infusions of rituximab, without the need for additional haematological chemotherapy, and the sustained complete remission of nephrotic syndrome after 2 years of follow-up, strongly suggest that the nephrotic-range proteinuria was mostly due to glomerular damage related to $\mathrm{MN}$ rather than to AL amyloid deposits.

In conclusion, optimal management of renal progression in patients with $\mathrm{AL}$ amyloidosis remains challenging after haematological treatment. Careful nephrological investigations, in addition to the standard haematological tests, are required in patients with successful treatment of $\mathrm{AL}$ amyloidosis and worsening of laboratory renal parameters. In such patients, kidney biopsy should be systematically discussed to investigate potential alternative glomerular diseases.

Acknowledgements: We thank Julie Sappa for editorial assistance.

Conflicts of interest and source of funding: Karim Belhadj reports personal fees from Celgene, personal fees from Amgen, personal fees from Takeda, and personal fees from Janssen, outside the submitted work. Vincent Audard reports personal fees from Addmedica outside the submitted work. Antoine Morel, David Buob, Jean-Michel Goujon, Marie-Christine Verpont and Anissa Moktefi report no conflicts of interest.

Antoine Morel ${ }^{1,2}$, David Buob ${ }^{3}$, Jean-Michel Goujon ${ }^{4,5}$, Karim Belhadj ${ }^{6,7}$, Marie-Christine Verpont ${ }^{8}$, Vincent Audard $^{1,2,7}$, Anissa Moktefi ${ }^{2,7,9}$

${ }^{1}$ Assistance Publique des Hôpitaux de Paris (AP-HP), Hôpitaux Universitaires Henri Mondor, Service de Néphrologie et Transplantation, Centre de Référence Maladie Rare Syndrome Néphrotique Idiopathique, Fédération Hospitalo-Universitaire, Innovative Therapy for Immune Disorders, Créteil, France; ${ }^{2}$ Université Paris Est Créteil, Institut National de la Santé et de la Recherche Médicale (INSERM) U955, Institut Mondor de Recherche Biomédicale (IMRB), Equipe 21, Créteil, France; ${ }^{3}$ Sorbonne Université, AP-HP, Service d'Anatomie et Cytologie Pathologiques, Hôpital Tenon, Paris, France; ${ }^{4}$ Département de Pathologie, Unité de Pathologie Ultrastructurale, CHU Poitiers, Poitiers, France; ${ }^{5}$ Centre de Référence Maladies Rares Amylose AL et Autres Maladies Par Dépôts D'immunoglobulines Monoclonales, Poitiers, France; ${ }^{6}$ AP-HP, Hôpitaux Universitaires Henri Mondor, Unité Hémopathies Lymphö̈des, Créteil, France; ${ }^{7}$ AP-HP, Hôpitaux Universitaires Henri Mondor, Réseau Amylose Mondor, Groupe de Recherche Clinique sur les Amyloses, Amyloid Research Institute, Créteil, France; ${ }^{8}$ Sorbonne Université, Plateforme d'Imagerie et de Cytométrie, UMRS, 1155, Paris, France; ${ }^{9} A P-H P$, Hôpitaux Universitaires Henri Mondor, Département de Pathologie, Créteil, France

Contact Dr Anissa Moktefi.

E-mail: anissa.moktefi@aphp.fr

1. Muchtar E, Dispenzieri A, Leung N, et al. Depth of organ response in $\mathrm{AL}$ amyloidosis is associated with improved survival: grading the organ response criteria. Leukemia 2018; 32: 2240-9.

2. Kaufman GP, Dispenzieri A, Gertz MA, et al. Kinetics of organ response and survival following normalization of the serum free light chain ratio in AL amyloidosis. Am J Hematol 2015; 90: 181-6.
3. Angel-Korman A, Jaberi A, Sanchorawala V, Avasi A. The utility of repeat kidney biopsy in systemic immunoglobulin light chain amyloidosis. Amyloid 2020; 27: 17-24.

4. Roth RM, Benson D, Hebert LA, et al. Progressive renal light chain amyloidosis with the absence of detectable free monoclonal light chains after an autologous hematopoietic stem cell transplant for amyloid light chain amyloidosis. Arch Pathol Lab Med 2013; 137: 1304-8.

5. Zeier M, Perz J, Linke RP, et al. No regression of renal AL amyloid in monoclonal gammopathy after successful autologous blood stem cell transplantation and significant clinical improvement. Nephrol Dial Transplant 2003; 18: 2644-7.

6. Sethi S. New 'antigens' in membranous nephropathy. J Am Soc Nephrol 2021; 32: 268-78.

7. Hoxha E, Wiech T, Stahl PR, et al. A mechanism for cancer-associated membranous nephropathy. N Engl J Med 2016; 374: 1995-6.

8. Troxell ML, Pilapil M, Miklos DB, et al. Renal pathology in hematopoietic cell transplantation recipients. Mod Pathol 2008; 21: 396-406.

9. Mrabet S, Aicha NB, Abdessayed N, et al. Membranous nephropathy succeeding autologous hematopoietic stem cell transplant: a case report. BMC Nephrol 2018; 19: 57.

10. Abudayyeh A, Truong LD, Beck Jr LH, et al. Membranous nephropathy in autologous hematopoietic stem cell transplant: autologous graftversus-host disease or autoimmunity induction? Clin Kidney J 2015; 8: 440-4.

11. Rubinstein S, Cornell RF, Du L, et al. Novel pathologic scoring tools predict end-stage kidney disease in light chain (AL) amyloidosis. Amyloid 2017; 24: 205-11.

12. Safadi S, Saad A, Quint PS, et al. Disappearance of immunoglobulins from persistent renal amyloid deposits following stem cell transplantation for heavy-and light-chain amyloidosis. Nephrol Dial Transplant 2015; 30: 1151-5.

DOI: https://doi.org/10.1016/j.pathol.2021.08.008

\section{Spindle cell lipoma with lipofibromatosis-like change: expanding the spectrum of morphological heterogeneity}

To the Editor,

Spindle cell lipoma (SCL) is a benign adipocytic neoplasm, first reported by Enzinger in $1975 .^{1}$ It most frequently occurs in the neck, shoulders and back region of middle-aged males. Histologically it is characterised by variable amounts of bland spindle cells, mature adipocytes, and ropy collagen bundles, described as thick, refractile and eosinophilic. ${ }^{2}$ Despite this characteristic triad of histological features, it is well recognised that SCL can display diverse morphological heterogeneity, and multiple pathological subtypes have been described. Indeed, the morphological features can show some overlap with other benign and malignant soft tissue tumours, presenting a potential diagnostic dilemma. In this report, we describe a case of SCL showing lipofibromatosis-like change, morphological changes that to our knowledge have not been reported in the literature to date.

A 48-year-old man presented with a mass localised to the left occiput. An ultrasound scan was performed revealing a lesion measuring $32 \times 29 \times 17 \mathrm{~mm}$ with features suggestive of a lipoma. A subsequent excision was performed by the patient's GP. Pathological examination of the specimen received showed pieces of yellow to tan tissue with a uniform appearance and cut surface and no evidence of haemorrhage or necrosis. Microscopically, the sections showed mature adipose tissue with lobularity and intervening incomplete fibrous septa composed of long sweeping fascicles of spindle cells. These cells contained bland, elongate and frequently 

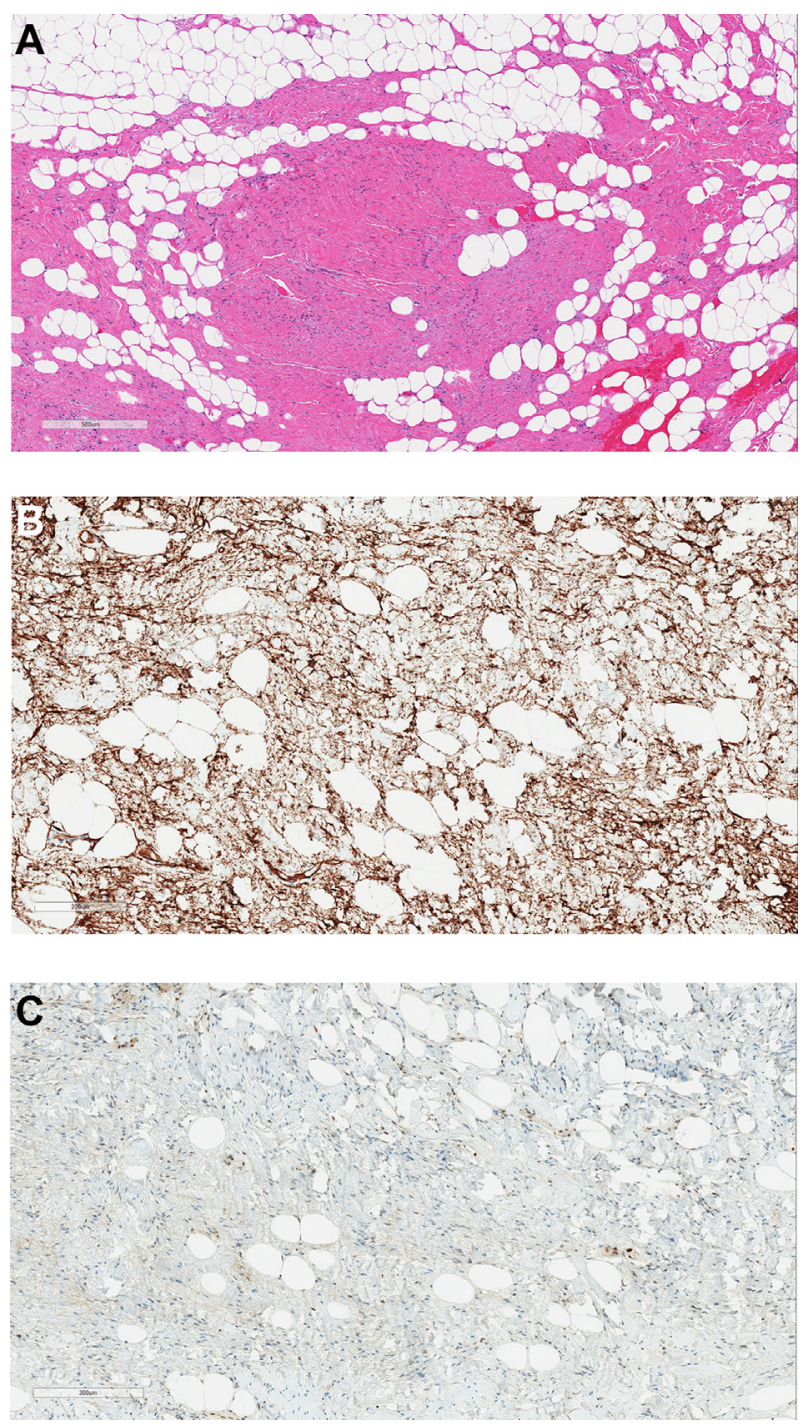

Fig. 1 (A) Spindle cell lipoma showing lipofibromatosis-like growth pattern (H\&E); (B) positive CD34 staining in the spindle cell component; (C) loss of nuclear staining with RB1.

buckled nuclei with no pleomorphism or hyperchromasia. These areas imparted a lipofibromatosis-like appearance (Fig. 1A). Further sections from the lesion were microscopically examined, leading to more conventional histological features, consistent with a diagnosis of SCL (Fig. 2).

Throughout the lesion, the spindle cells showed positive cytoplasmic staining with CD34 (Fig. 1B). Focal weak

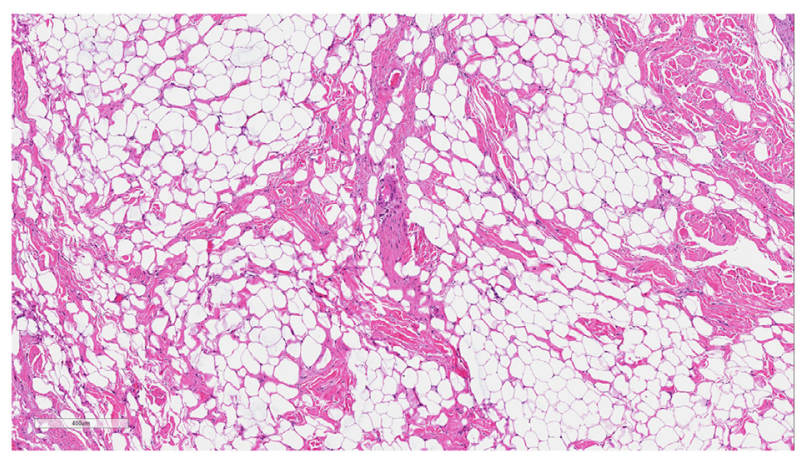

Fig. 2 Spindle cell lipoma with conventional morphological features (H\&E). nuclear staining was present with S100. There was loss of nuclear staining with RB1 in both the lipofibromatosis-like areas as well as the conventional SCL areas (Fig. 1C). Negative staining was seen with both $\beta$-catenin and PANTRK antibodies.

SCL is a benign adipocytic tumour which accounts for approximately $1.5 \%$ of all adipocytic neoplasms. ${ }^{2}$ It most commonly occurs in middle aged males with $<10 \%$ of cases occurring in female patients. Approximately $80 \%$ of cases arise within the subcutis of the posterior neck, back and shoulders, however various other locations are well described in the literature. ${ }^{3}$ Conservative excision is usually adequate treatment. Metastases do not occur and local recurrence is rare, even in the setting of incomplete excision. ${ }^{2}$

Histologically, SCL is characterised by a triad of features including bland spindle cells, mature adipocytes and ropy collagen bundles. Despite these typical features, there are numerous morphological variants of SCL described in the literature including but not limited to the myxoid, fat-poor, (pseudo)angiomatous and plexiform subtypes in addition to SCL with focal cartilaginous and osseous metaplasia and SCL with extramedullary haematopoiesis. Indeed, SCL also lies on a morphological spectrum with pleomorphic lipoma (PL) which is characterised by pleomorphic spindle cells and floret-like giant cells that characteristically stain positive for CD34. ${ }^{2}$

Both SCL and PL are characterised by partial or whole chromosome 13 and/or 16 deletions. Described breakpoints in $13 q$ deletions cluster around the region $13 q 14$, where the RB1 gene is located. ${ }^{4}$ For this reason, immunohistochemical staining for RB1 can act as a surrogate marker in the diagnosis of SCL/PL. Loss of RB1 can be especially helpful in making the diagnosis of SCL in the setting of variant morphology, as illustrated in this case.

In this case, we describe a rare variant of SCL showing lipofibromatosis-like changes, a novel morphological finding in the setting of SCL. Lipofibromatosis is a rare soft tissue neoplasm affecting the paediatric population, with half of all cases being diagnosed before the age of 12 months. Up to $20 \%$ of cases are congenital. First described in 2000, lipofibromatosis presents as a slow-growing subcutaneous nodule in the distal extremities, particularly the hands and feet, ${ }^{5}$ with an even distribution among male and female patients. ${ }^{6}$ Although lipofibromatosis has no known metastatic potential, it may display locally aggressive features including diffuse and infiltrative growth patterns. Local recurrence is seen in up to $70 \%$ of cases.

A diagnosis of lipofibromatosis can only be made on histological grounds. It is characterised by mature adipose tissue traversed by cellular fascicles of spindle cells with bland, uniform, elongated nuclei, features similar to those seen in our case. The immunohistochemical profile is non-specific with spindle cells showing variable expression of CD34 and SMA and negative staining with desmin. Molecular studies have identified several gene fusions encoding receptor tyrosine kinases or their ligands, suggesting a shared deregulation of the PI3K-AKT-mTOR pathway which may be implicated in its pathogenesis. ${ }^{8}$ Despite this, no robust or reproducible molecular target has been identified to date.

Also to be considered in the differential diagnosis is lipofibromatosis-like neural tumour (LPF-NT), an entity that exists within the emerging spectrum of NTRK-rearranged spindle cell neoplasms, included in the most recent edition 
of the World Health Organization (WHO) classification. ${ }^{9}$ This is a rare soft tissue neoplasm, with the majority of cases occurring in the first two decades of life, however it has also been described in middle aged adults. ${ }^{10}$ They can present as superficial or deep tumours in the extremities or trunk region. Benign LPF-NT show a significant rate of recurrence, owing to the infiltrative growth pattern seen histologically, with no metastases described. However, metastasis has been reported in tumours showing high-grade morphological features.

NTRK-rearranged spindle cell neoplasms are an evolving entity within which a spectrum of morphological features are seen. LPF-NT is characterised histologically by haphazardly arranged fascicles of spindle cells that show an infiltrative growth pattern within subcutaneous fat, resembling lipofibromatosis. The cells contain monomorphic nuclei with tapering poles and indistinct cytoplasm. Most cases of LPFNT show co-expression of CD34 and S100, as in this case. Most tumours characteristically show NTRK1 fusions, although small numbers of cases showing NTRK2 or NTRK3 fusions have also been described. ${ }^{9}$ These gene fusions underpin the oncogenic activation of downstream tyrosine kinase proteins. ${ }^{11}$ Thus, the majority of these lesions are reactive with an anti-pan-TRK monoclonal antibody.

In summary, we describe a case of a SCL showing lipofibromatosis-like changes. These changes can lead to a differential diagnosis including SCL, lipofibromatosis and LPF-NT. The clinical setting and immunohistochemical findings (particularly loss of RB1 nuclear staining and negative staining with PAN-TRK) is consistent with a diagnosis of SCL. In addition, the examination of further tissue sections and finding of more conventional morphological features of SCL strengthens the diagnosis. This is an important distinction owing to the increased risk of recurrence and aggressive local growth associated with lipofibromatosis and LPF-NT. Pathologists should be conscious of the morphological spectrum seen in SCL, taking care to correlate with the clinical setting and immunohistochemical findings in order to avoid misdiagnosis.

Conflicts of interest and sources of funding: No funding was received for this work. The authors state that there are no conflicts of interest to disclose.

\section{P. O’Neill ${ }^{1}$, S. Pirasteh ${ }^{2}$, D. Paton ${ }^{2}$}

${ }^{1}$ PathWest Anatomical Pathology, QEII Medical Centre, Nedlands, WA, Australia; ${ }^{2}$ Western Diagnostic Pathology, Histopathology, Jandokot, WA, Australia

Contact Dr Paul O’Neill.

E-mail: poneill22@qub.ac.uk

1. Enzinger FM, Harvey DA. Spindle cell lipoma. Cancer 1975; 36: 1852-9

2. Billings SD, Ud Din N. Spindle cell lipoma and pleomorphic lipoma. In: WHO Classification of Tumours Editorial Board. World Health Organization Classification Tumours. Soft Tissue and Bone Tumours. 5th ed. Lyon: IARC, 2020; 29-30.

3. Chen S, Huang H, He S, et al. Spindle cell lipoma: clinicopathologic characterization of 40 cases. Int J Clin Exp Pathol 2019; 12: 2613-21.

4. Dahlén A, Debiec-Rychter M, Pedeutour F, et al. Clustering of deletions on chromosome 13 in benign and low-malignant lipomatous tumors. Int $J$ Cancer 2003; 20: 616-23.

5. Fetsch JF, Miettinen M, Laskin WB, et al. A clinicopathologic study of 45 pediatric soft tissue tumors with an admixture of adipose tissue and fibroblastic elements, and a proposal for classification as lipofibromatosis. Am J Surg Pathol 2000; 24: 1491-500.

6. Boos MD, Chikwava KR, Dormans JP, et al. Lipofibromatosis: an institutional and literature review of an uncommon entity. Pediatr Dermatol 2014; 31: 298-304.

7. Miettinen M, Al-Ibraheemi A, Zambrano E. Lipofibromatosis. In: WHO Classification of Tumours Editorial Board. World Health Organization Classification Tumours. Soft Tissue and Bone Tumours. 5th ed. Lyon IARC, 2020; 96-7.

8. Al-Ibraheemi A, Folpe AL, Perez-Atayde AR, et al. Aberrant receptor tyrosine kinase signaling in lipofibromatosis: a clinicopathological and molecular genetic study of 20 cases. Mod Pathol 2019; 32: 423-34.

9. Suurmeijer AJH, Antonescu CR. NTRK-rearranged spindle cell neoplasm (emerging). In: WHO Classification of Tumours Editorial Board. World Health Organization Classification Tumours. Soft Tissue and Bone Tumours. 5th ed. Lyon: IARC, 2020; 287-9.

10. Crumbach L, Descotes F, Bringuier PP, et al. Lipofibromatosis-like neural tumor: a case report and review of the literature. Am J Derma topathol 2020; 42: 881-4.

11. Cocco E, Scaltriti M, Drilon A. NTRK fusion-positive cancers and TRK inhibitor therapy. Nat Rev Clin Oncol 2018; 15: 731-47.

DOI: https://doi.org/10.1016/j.pathol.2021.09.014

\section{Local genomic sequencing enhances COVID-19 surveillance in the Northern Territory of Australia}

\section{To the Editor,}

Genomic sequencing has played a critical role in the global public health response to COVID-19. Within days of the first reported cases of pneumonia of unknown aetiology in Wuhan, the novel coronavirus now known as SARS-CoV-2 was identified by metagenomic sequencing and made publicly available, enabling rapid development of sensitive and specific diagnostic tests and development of effective vaccines. ${ }^{1}$ Genomic sequencing is now a gold standard component of 'precision public health', with surveillance applications including outbreak investigation, identification of emerging variants of concern, and estimation of transmission dynamics. $^{2-4}$ In 2020, the AusTrakka platform developed by the Communicable Disease Genomics Network was launched for data sharing and centralised pathogen genomic analysis across Australia and New Zealand, ${ }^{5}$ and all jurisdictions now have capacity to sequence and submit SARS-CoV-2 genomes.

SARS-CoV-2 variants of concern associated with increased transmissibility, increased virulence, and/or immune evasion, have been defined by the World Health Organization (WHO). In April-May 2021, SARS-CoV-2 lineage B.1.617.2 (Delta) caused a surge in cases in India, subsequently becoming the dominant SARS-CoV-2 lineage globally. ${ }^{6}$ In late November 2021 there was a rapid increase in SARS-CoV-2 lineage B.1.1.529 case numbers in Gauteng province, South Africa, displacing Delta as the dominant strain. ${ }^{7}$ B.1.1.529 is phylogenetically distinct from other variants of concern and has many mutations predicted to be associated with immune evasion and increased transmissibility. ${ }^{8}$ This lineage was designated the Omicron variant of concern by WHO on 26 November 2021.

The Northern Territory (NT) is a sparsely populated and geographically remote Australian jurisdiction, with a population of 245,000 and an area of over a million square kilometres. Approximately one-third of the population are Australian First Nations people, almost three-quarters of whom live in remote regions. ${ }^{9}$ To mitigate the risk of 